Paidéia, 2002, 12(23), 105-112

\title{
AVALIAÇÃO COGNITIVA DE CRIANÇAS COM DIFICULDADES DE APRENDIZAGEM: PRECISÃO DO TESTE DE GOODENOUGH (1926) E DA EMMC (1993) ${ }^{1}$
}

\author{
Susi Lippi Marques ${ }^{2}$ \\ Universidade Federal de São Carlos \\ Sonia Regina Pasian \\ Universidade de São Paulo RP \\ Maria Aparecida Paiva Franco \\ Ivana Regina Panosso \\ Araguacy Brazil Viana \\ Daniela Andrea de Oliveira \\ Universidade Federal de São Carlos
}

\begin{abstract}
RESUMO: A confiabilidade nos resultados dos testes intelectuais tem sido investigada através de teste-reteste com breves intervalos de tempo, questionando-se sua efetiva estabilidade diante das mudanças sócio-culturais das últimas décadas. Neste estudo investigou-se a estabilidade temporal, após longo intervalo entre teste-reteste, do Teste de Goodenough (1926/1964) e da Escala de Maturidade Mental Colúmbia (EMMC, 1972/1993). Foram avaliados 60 alunos (ambos os sexos) de escola pública de ensino fundamental de sete a nove anos, com dificuldades de aprendizagem. Os testes foram aplicados, individualmente, na própria escola, com intervalo de seis meses entre teste-reteste. Foi encontrado índice de correlação de 0.631 entre pontos brutos nas duas aplicações do Teste de Goodenough. Em relação a EMMC (1972/1993) foi encontrado índice de correlação de 0.608 entre resultados padrão por idade das duas testagens. Os resultados apontaram razoável estabilidade dos dados, oferecendo confiabilidade técnica na avaliação cognitiva infantil.
\end{abstract}

Palavras-chave: Teste de Goodenough, Escala de Maturidade Mental Colúmbia, Fidedignidade

\section{RELIABILITY OF GOODENOUGH TEST (1926) AND CMMS (1993) in COGNITIVE ASSESSMENT OF CHILDREN with learning difficulties}

\begin{abstract}
The reliability of intellectual test results has been investigated by test-retest assessment at short intervals, which leads to doubts about its effective stability, mainly because of the intensity and quickness of socio-cultural and environmental changes in the last decades. This study has aimed at investigating the temporal stability, after a long interval between test-retest, through Goodenough Test (1926/1964) and the Columbia Maturity Mental Scale (CMMS, 1972/1993). We have assessed 60 students (both sexes) at a primary state school, from seven to nine years old, presented learning difficulties. The tests were applied, individually, in two occasions, with an interval of six months between test-retest. It was found a correlation index of 0.631 between the whole scores obtained in two Goodenough Tests (1926/1964) applied. Concerning the CMMS (1972/1993) it was found a correlation index of 0.608 between the age standard results. The indexes obtained have suggested reasonable stability of data, offering technical reliability of children's cognitive assessment.
\end{abstract}

Key-words: Goodenough Test, Columbia Maturity Mental Scale (CMMS), Reliability,

\footnotetext{
${ }^{1}$ Artigo recebido para publicaçăo em fevereiro de 2002; aceito em majo de 2002

${ }^{2}$ Endereço para correspondência: Susi Lippi Marques Oliveira, Centro de Educação e Ciências Humanas, Departamento de Psicologia, Universidade Federal de São Carlos, Rodovia Washington Luis (SP-310), Km 235, São Carlos, SP, Cep 13565-905, E-mail susilmo@power.ufscar.br
} 
A utilização de produções gráficas, em diferentes faixas etárias, tem sido histórica e amplamente reconhecida como estratégia útil e relevante para $o$ acesso a marcas do processo de desenvolvimento e da funcionalidade dos indivíduos em suas diferentes atividades no contato com o ambiente, incluindo funções psicomotoras, cognitivas, emocionais e mesmo neurológicas. A literatura científica relativa a esta temática, além de muito extensa, perpassa os séculos na busca de indicadores técnicos qualificadores do desenvolvimento e das potencialidades humanas em suas diferentes etapas de vida. Nesta perspectiva, amplas revisões teóricas sobre o tema foram realizadas, disponibilizando argumentos favoráveis e contrários a estas estratégias de avaliação psicológi$\mathrm{ca}$, sem conclusões definitivas sobre o tema. Apesar disso, como comentam Abell, Horkheimer e Nguyen (1998) ao estudar métodos de avaliação intelectual através de Desenhos de Figura Humana (DFH) em adolescentes, estes instrumentos possuem uma longa história de uso pelos psicólogos como técnicas úteis e preditoras da habilidade cognitiva e do desempenho acadêmico, aspecto também reiterado no estudo de Aikman, Belter e Finch (1992). Dada esta popularidade mundial destes instrumentos, muitos sistemas de avaliação dos DFH foram desenvolvidos para acessar habilidades cognitivas, com destaque para o trabalho pioneiro de Goodenough (1926/ 1964), depois revisto por Harris, transformando-se provavelmente no sistema mais corhecido para esta finalidade (Goodenough-Harris, 1963), conforme apontam Abell, Horkheimer e Nguyen (1998).

Dentro da multiplicidade de argumentos teóricos e evidências empíricas existentes na literatura relativa ao $\mathrm{DFH}$, há relativo consenso desta sua utilidade para oferecer elementos sobre a maturidade do desenvolvimento conceitual dos indivíduos, nomeadamente com crianças. Questiona-se sobre a interferência da habilidade artística, a percepção visual e os padrốes de coordenação motora como variáveis de interferência no processo expressivo e avaliativo por meio de técnicas gráficas, em especial pelo DFH, como apontam Aikman, Belter e Finch (1992). Apesar dessas interferências, reconhece-se o "desempenho gráfico infantil enquanto manifestação aplicada e dinâmica de competências intelectuais, encaradas na sua funcionalidade (...), permi- tindo acompanhar o desenvolvimento de uma organização mental." (Santos, Colaço, Taborda \& Alvarez, 1997).

Neste processo avaliativo por meio de técnicas de exame psicológico, fazem-se necessários cuidados metodológicos com os instrumentos a serem utilizados, buscando-se índices válidos e precisos sobre a função psicológica em investigação. Esta mesma preocupação ocorre quando se recorre ao DFH como estratégia de acesso à maturidade conceitual dos indivíduos. Historicamente, como já apontado, a prática clínica dos psicólogos instituiu esta técnica como válida, persistindo, no entanto, preocupações permanentes com a fidedignidade dos índices tomados a partir dos DFH, considerando-se a diversidade de sistemas avaliativos desenvolvidos. A meta seria alcançar indicadores precisos sobre a função examinada no indivíduo, de modo que as possibilidades de imperfeições neste acesso a seu dinamismo psíquico fossem mínimas, considerando a impossibilidade de sua inexistência na prática. Assim, retomamos com Burgemeister, Blum e Lorge (1993) a definição do conceito de fidedignidade:

\begin{abstract}
"A precisão do teste - ou o grau em que os escores do teste para um dado grupo refletem diferenças individuais 'verdadeiras', livres de erros de medida - é usualmente expressa como um coeficiente de correlação chamado, mais exatamente, coeficiente de precisão." (p. 5I)
\end{abstract}

Múltiplos e recorrentes esforços foram desenvolvidos nas várias décadas de utilização dos DFH como instrumentos de avaliação psicológica, enfatizando-se a necessidade de parâmetros avaliativos válidos, precisos e adequados ao padrão sócio-cultural dos indivíduos (Anastasi \& Urbina, 2000). Em nosso país estas tentativas também existiram e permanecem como aspecto relevante na pesquisa da área de avaliação psicológica; podendose destacar os trabalhos de melhoria dos instrumentos utilizados para avaliar o desenvolvimento cognitivo de crianças brasileiras desenvolvidos por Alves (1979), Alves e Duarte (1993), Hutz e Antoniazzi (1995), Wechsler (1996) e Sarti (1999), entre outros.

Tentativas de exame da confiabilidade das técnicas de exame psicológico cognitivo infantil, especificamente dirigidas ao estudo do Teste de 
Goodenough (1926/1964) e a Escala de Maturidade Mental Colúmbia - EMMC (1972/1993) foram também desenvolvidas desde sua criação até recentemente, reiterando a possibilidade de uso destes instrumentos com estes objetivos. Dentre a diversidade destes trabalhos, apenas para caráter ilustrativo dos parâmetros de confiabilidade destas técnicas, a própria Goodenough encontrou um índice de correlação teste-reteste de 0.937 com crianças, enquanto, no Brasil, Alves (1979) descreveu vários trabalhos que chegaram a índices de 0.650 a 0.957 , também com população infantil. Confirmam-se, portanto, indicadores da precisão deste instrumento como medida da maturidade conceitual infantil. Já com a EMMC (1972/1993), os autores da técnica apontaram índices de correlação teste-reteste variando entre 0.84 a 0.86 (Burgemeister, Blum \& Lorge, 1972/1993), ao passo que Alves e Duarte (1993), ao padronizarem o teste para o Brasil, encontraram índices de precisão teste-reteste entre 0.73 a 0.90 . Confirma-se, desta forma, a fidedignidade também da EMMC como indicador da maturidade mental de crianças. Há que se fazer a ressalva de que nestes estudos sobre a precisão destes instrumentos através do método teste-reteste houve a utilização de amostras muito diversificadas, abordando indivíduos de diferentes faixas etárias e características clínicas, além de variados intervalos de tempo entre teste e reteste (embora, no geral, de um a dez dias), limitando a possibilidade comparativa entre os resultados dos vários estudós.

Mesmo após estes esforços, os processos de avaliação cognitiva infantil por meio de $\mathrm{DFH}$ continuam apresentando variações em seus resultados, sobretudo em função do sistema avaliativo utilizado, dificultando a estabilidade destes procedimentos, embora recorrentes na prática clínica, sobretudo em ambientes escolares. A precisão do diagnóstico psicológico de crianças, do ponto de vista de maturidade conceitual, sofre, então, variações processuais relacionadas ao método de análise da produção gráfica, dificultando comparações e a confiabilidade nos resultados obtidos.

Na prática cotidiana de avaliação psicológica, embora existam os estudos técnicos que buscaram aprimorar os métodos de análise do $\mathrm{DFH}$, como elaborado por Alves (1979), Hutz e Antoniazzi
(1995), Wechsler (1996) e Sarti (1999), nem sempre são utilizados os procedimentos mais recentemente padronizados e pesquisados para a nossa realidade sócio-cultural, como deveria ocorrer. Muitas vezes os profissionais acabam por utilizar o material ou o sistema avaliativo do DFH em que foram treinados em sua graduação em Psicologia, sem acompanhar os avanços técnicos existentes na área. Exemplo dessa situação é a frequiente recorrência ao uso do Teste de Goodenough em nossas escolas, apesar da existência dos outros sistemas avaliativos já referidos e otimizados para o mesmo objetivo.

Considerando-se esta realidade existente no cotidiano da prática profissional em nossa realidade, faz-se necessário estudar também as propriedades psicométricas do Teste de Goodenough na atualidade, procurando buscar elementos técnicos esclarecedores sobre suas possibilidades informativas a respeito do desenvolvimento cognitivo infantil, em termos conceituais. Para sustentar o seu uso, seria necessário reconhecer a qualidade de seus indicadores técnicos, nomeadamente à questão relativa aos índices de confiabilidade nos indicadores obtidos a partir deste instrumento de avaliação psicológica, aspecto de extrema relevância técnica.

Uma das formas de estudo da precisão de uma medida psicológica é a comparação de resultados de um indivíduo em dois momentos avaliativos, a partir de mesmos instrumentos (Anastasi, 1976; Burgemeister, Blum \& Lorge 1993; Anastasi \& Urbina, 2000). A contraposição de avaliações (testereteste) com breves intervalos de tempo, sendo comum períodos de um a dez dias para se realizar a retestagem, tem sido freqüentemente utilizada para se investigar a estabilidade dos resultados dos testes psicológicos. Isso tende a propiciar questionamento sobre a efetiva estabilidade das informações obtidas, sobretudo diante da intensidade e rapidez das mudanças sócio-culturais e ambientais das últimas décadas (Anastasi, 1976; Anastasi \& Urbina, 2000; Cunha \& cols., 2000). Como ficariam os dados sobre a maturidade cognitiva infantil obtidos por meio do Teste de Goodenough e por meio da EMMC após intervalos maiores de tempo entre teste e reteste? Permaneceriam nos mesmos parâmetros, atestando confiança à medida tomada inicialmente, ou refletiriam variações suscitadoras de questionamenio 


\section{Susi Lippi Marques}

de suas informações sobre o potencial cognitivo infantil? Serviriam mais para retratos situacionais de desempenho das crianças e não para indicadores de capacidade e maturidade conceitual das mesmas?

Fctas questões, dentro dos limites de acesso bibliográfico possível, não pareceram foco recente de investigação em nossa realidade, apesar do intenso uso do Teste de Goodenough como medida do potencial cognitivo infantil. Santos, Colaço, Taborda e Alvarez (1997) realizaram um estudo de.DFH através do sistema avaliativo de Goodenough, procurando examinar quais as qualidades e as limitações dos índices obtidos por meio desta técnica. Estudaram 121 crianças portuguesas de 6 a 10 anos, do ensino básico, encontrando índices de precisão (pelo método da consistência interna) bastante encorajadores, como por eles referido no artigo. Em nossa realidade, Alves (1979) estudou crianças préescolares por meio do $\mathrm{DFH}$, comparando os resultados obtidos pelo sistema avaliativo de Goodenough e de Goodenough-Harris (1963). Concluiu que seria mais adequada a utilização deste segundo método de análise do DFH, inclusive apresentando normas preliminares para avaliação de pré-escolares da região de São Paulo. Contudo, na prática clínica cotidiana, deparamo-nos freqüentemente com a recorrência do Teste de Goodenough (1926/1964), justificando-se, assim, investimentos de análise de suas possibilidades informativas em nossa realidade atual.

Considerando este freqüente uso do Teste de Goodenough e da EMMC em nosso cotidiano e a existência de reduzida investigação sobre a estabilidade de seus resultados, sobretudo a partir de intervalos maiores de tempo entre teste e reteste, o presente trabalho objetivou examinar os índices de fidedignidade do DHF pelo sistema de Goodenough (1926) e da EMMC (1993) após um semestre da avaliação inicial de crianças escolares do ensino fundamental. Almejou-se, portanto, investigar em que medida estas provas seriam estáveis em termos de sua proposta básica, ou seja, avaliação da inteligência, enquanto capacidade conceitual e enquanto índice de maturidade mental infantil. Focalizou-se o índice de estabilidade das medidas cognitivas obtidas por cada uma delas, mesmo após um longo e significativo intervalo de tempo (seis meses), objetivando examinar se os indicadores técnicos resistiriam a esta variável temporal e às possibilidades naturais do processo de maturação infantil intrínsecas neste período.

\section{Método}

\section{Participantes}

A investigação foi realizada em uma escola estadual de ensino fundamental (primeira a quarta séries) da cidade de São Carlos (SP), localizada numa região considerada de exclusão social, onde há grande disseminação de pobreza. De acordo com as informações dadas pela diretora da escola, a maioria dos pais dos alunos eram analfabetos. Participaram do estudo 60 alunos de sete a nove anos, de ambos os sexos (42 meninos e 18 meninas), sendo 33 alunos da primeira série, 10 alunos da segunda série e 17 da terceira série.

Considerando-se que as crianças deste estudo fariam parte de um projeto maior de investigação da eficácia de programas de apoio a problemas de aprendizagem desenvolvidos e a ser implementados por uma equipe de profissionais do Departamento de Psicologia da Universidade Federal de São Carlos - UFSCar, os participantes deste trabalho foram crianças indicadas pelas respectivas professoras como possuidoras de dificuldades de aprendizagem. Esta indicação dos alunos com problemas no rendimento acadêmico foi realizada após seis meses do início do ano letivo.

\section{Material}

Neste estudo foram utilizados os materiais básicos para aplicação, registro e avaliação das específicas técnicas de exame psicológico aplicadas, a saber: a) folha de papel sulfite, lápis preto e borracha (para o Teste de Goodenough); b) conjunto de cartões da EMMC; c) folhas de registro e de avaliação da produtividade das crianças nas duas técnicas; d) manuais do Teste de Goodenough e da EMMC para análise e comparação normativa dos dados; e) cronômetro; f) termo de consentimento livre e esclarecido para a pesquisa, oferecido aos pais e/ou responsáveis pelas crianças participantes do estudo.

\section{Procedimento}

Após os devidos cuidados de solicitação de consentimento para a pesquisa por parte da instituição escolar e dos pais e/ou responsáveis pelas crianças, os alunos selecionados (indicados pelas respectivas 
professoras como possuidores de dificuldades de aprendizagem, a partir do desempenho obtido no primeiro semestre letivo de sua série escolar) foram convidados a participar deste estudo por uma das autoras (graduandas em Psicologia, com prévio treinamento técnico nos instrumentos e com supervisão docente). Caso a criança concordasse, era submetida à aplicação individual, padronizada, do Teste de Goodenough e da EMMC, após estabelecimento de adequado rapport, em uma sala apropriada para avaliação psicológica nas dependências da própria escola, durante o seu período de aulas.

\section{Resultados e Discussão}

Considerando-se os objetivos propostos, inicialmente serão apresentados os dados relativos aos índices de fidedignidade (teste-reteste) obtidos, de modo independente, para as duas técnicas de avaliação psicológica utilizadas. A análise dos resultados foi feita com base nos 41 alunos que permaneceram até o final do estudo, comparando-se suas produções no Teste de Goodenough, em termos de pontos brutos obtidos, e na EMMC, através dos resultados padrão por idade (RPI). A análise da produtividade nestas técnicas foi feita separadamente com objetivo de checar a estabilidade temporal de seus resultados e possibilidades informativas após um intervalo de seis meses, considerado bastante extenso em termos do desenvolvimento infantil.

A comparação entre os resultados obtidos (pontos brutos, considerando-se a inexistência de normas brasileiras atualizadas e adequadas para as características da amostra avaliada) nas duas ocasiões de aplicação da Escala de Goodenough foi investigada pelo índice de correlação de Pearson. Elaborou-se também uma estatística descritiva básica (média, desvio-padrão, mediana e erro padrão de medida $=E P M)$ do desempenho dos 41 escolares no DFH, conforme apresentado na seguinte Tabela 1.

Tabela 1: Estatística descritiva do desempenho de crianças escolares $(n=41)$ do ensino fundamental no Teste de Goodenough e respectivo índice de correlação entre pontos brutos do teste e do reteste (após seis meses)

\begin{tabular}{cccccccccc}
\hline Partici- & Correlação & \multicolumn{2}{c}{ Média } & \multicolumn{2}{c}{ D.P. } & \multicolumn{2}{c}{ Mediana } & \multicolumn{2}{c}{ E.P.M. } \\
pantes & teste-reteste* & Teste & Reteste & Teste & Reteste & Teste & Reteste & Teste & Reteste \\
\hline 41 & 0.631 & 17.47 & 18.54 & 4.45 & 7.06 & 18 & 16 & 0.57 & 1.10 \\
\hline$* \mathrm{p}<0.01$ & & & & & & & & &
\end{tabular}

Embora exista variação nos resultados encontrados em ambos os momentos avaliativos e um desempenho gráfico sinalizador de alguma dificuldade cognitiva (resultados médios estão levemente inferiores à faixa de normalidade), a correlação encontrada entre teste-reteste atingiu bom índice (correlação de Pearson = 0.631), mesmo após meio ano na vida destas crianças.

Realizando-se uma análise estatística comparativa (por meio do Teste $t$ de Student para amostras correlacionadas) dos desempenhos obtidos pelas 41 crianças avaliadas no Teste de Goodenough, não foram encontradas diferenças entre teste e reteste (porque $\mathrm{P}$ >
0.50). Confirmou-se, através também deste procedimento, a estabilidade do desempenho cognitivo por meio deste instrumento de avaliação psicológica infantil.

$\mathrm{O}$ mesmo procedimento analítico foi desenvolvido para os dados colhidos com a EMMC, respeitando-se sua especificidade técnica de apresentação de desempenhos das crianças em resultados padrão por idade (RPI). Neste instrumento avaliativo a faixa de normalidade da maturação cognitiva infantil abrange de 84 a 111 pontos no RPI. Os resultados obtidos com a EEMC nos 41 escolares avaliados, bem como a análise comparativa de seus desempenhos no teste $\mathrm{e}$ no reteste, podem ser vistos na Tabela 2.

Tabela 2: Estatística descritiva do desempenho de crianças escolares $(n=41)$ do ensino fundamental na Escala de Maturidade Mental Colúmbia e respectivo índice de correlação entre resultados padrão por idade (RPI) do teste e do reteste (após seis meses) 


\section{Susi Lippi Marques}

Novamente encontra-se alguma variação entre os desempenhos infantis nos dois momentos avaliativos, porém agora mais favoráveis desde a primeira testagem, com sinais de adequação da maturidade intelectual do conjunto das crianças avaliadas. Este dado, em si, ilustra a importância e a relevância dos estudos de padronização das técnicas de exame psicológico, dado isto ter sido desenvolvido recentemente para com a EMMC (1972/1993) em nosso meio (Alves \& Duarte, 1993), atestando a necessidade de parâmetros normativos atualizados para os processos psicodiagnósticos.

A análise estatística comparativa dos desempenhos do conjunto de escolares entre a primeira e segunda aplicação da EMMC (por meio do teste t de Student para amostras correlacionadas), embora aparentemente evidencie alguma variação nos resultados (com aumento do RPI na segunda avaliação), não sinalizou diferença significativa entre os mesmos (porque $\mathrm{P}>0,50$ ). Este dado pode ser compreendido como confirmador da hipótese de estabilidade do desempenho cognitivo destas crianças, mesmo após longo intervalo de tempo, dentro das informações disponibilizadas pela EMMC.

Reiterando estas evidências, o índice de correlação obtido entre teste-reteste (correlação de Pearson $=0.608$ ) neste instrumento de avaliação psicológica alcançou valor sugestivo de razoável precisão da EMMC, evidenciando estabilidade dos resultados após seis meses da primeira testagem. Este dado aponta que as informações sobre o índice de maturidade cognitiva obtido por meio desta prova é confiável ao longo do desenvolvimento do ano letivo destas crianças avaliadas, mesmo com suas dificuldades de rendimento escolar. Ou seja, apesar do tempo e das múltiplas variáveis intervenientes nesses seis meses de intervalo entre a primeira e a segunda avaliação psicológica, os resultados na EMMC foram bastante similares nestes dois momentos, oferecendo confiabilidade ao índice de maturidade cognitiva possível de ser obtido por meio desta técnica não verbal de avaliação da inteligência infantil.

Considerou-se de interesse, neste trabalho, ainda analisar e rapidamente refletir sobre a peculiaridade de se examinar a estabilidade do desempenho cognitivo em crianças com dificuldades de aprendizagem, aspecto dilemático na prática cotidiana do psicólogo escolar. Os atuais resultados da EMMC, obtidos com escolares com problemas de rendimento acadêmico, evidenciaram um coeficiente de correlação entre teste-reteste igual a 0.608 , considerado adequado índice de confiabilidade para a avaliação realizada. Comparando-o com o índice obtido no estudo de padronização da EMMC por Alves e Duarte (1993), em um reteste realizado após sete a dez dias do teste com crianças sem dificuldades de aprendizagem, encontramos dados que merecem cautelosa consideração na prática psicodiagnóstica. Especificando esta análise, Alves e Duarte (1993) encontraram um coeficiente de precisão de teste-reteste mediano de 0.85 (Burgemeister, Blum \& Lorge, 1972/ 1993). Sendo assim, os índices apresentados neste trabalho apontaram razoável estabilidade dos dados obtidos com a EMMC, porém com um índice inferior ao encontrado em reavaliações com menores intervalos de tempo e com crianças sem dificuldades de aprendizagem. Ou seja, apesar das atuais evidências empíricas atualizadas de confiabilidade técnica na avaliação cognitiva por meio da EMMC (1993) dentro da nossa realidade sócio-cultural, será preciso levar em consideração, na análise dos desempenhos infantis, as variáveis evolutivas e do rendimento escolar, pois sinalizaram relevante influência no padrão geral de desempenho nesta técnica. Estes elementos, embora aparentemente óbvios, podem ser pouco considerados na prática clínica cotidiana, podendo induzir hipóteses interpretativas indevidas pelos utilizadores destes instrumentos de avaliação cognitiva infantil, razão maior desta breve consideração reflexiva e comparativa de estudos sobre a fidedignidade da EMMC.

Os resultados encontrados neste estudo apontaram para bons índices de fidedignidade para o sistema avaliativo de Goodenough e para a Escala de Maturidade Mental Colúmbia (1972/1993) após um intervalo de seis meses, qualificando-os como estratégias confiáveis para a avaliação da capacidade cognitiva infantil na realidade sócio-cultural contemporânea.

As evidências empíricas apresentadas neste trabalho, relativas à confiabilidade dos referidos instrumentos de avaliação psicológica, depreendidos a partir do desempenho de crianças com dificuldades de aprendizagem assumem, de nosso ponto de vista, 
especial relevância em nosso contexto cotidiano de trabalho em avaliação psicológica, onde verificamos freqüentemente o uso do Teste de Goodenough (1926/1964). Apesar deste método ter sido elaborado há longa data e ter sofrido reformulações significativas (Goodenough-Harris, 1963; e, em nosso contexto, Wechsler, 1996), evidenciou propriedades que ainda sustentam sua utilidade e possibilidade informativa, dentro de seus limites técnicos. O mesmo poderia ser comentado para os resultados obtidos com a EMMC (1972/1993) que, embora recentemente padronizada para o Brasil (Alves e Duarte, 1993), não havia sido examinada com relação a crianças com problemas no rendimento acadêmico. Os índices encontrados no presente trabalho possibilitam a utilização confiável deste procedimento mesmo nestes casos, apresentando base empírica adicional para a investigação da maturidade conceitual infantil.

Cabe ainda realizar uma breve análise comparativa entre os clássicos estudos de fidedignidade realizados com o Teste de Goodenough e com a EMMC diante dos dados obtidos neste estudo. Obviamente este processo de tentativas comparativas precisa ser adequadamente dimensionado em seus limites informativos, em função das múltiplas diferenças entre os trabaihos realizados (características das amostras, épocas dos trabalhos, intervalos testereteste, entre outras). Dentro desta realidade, observamos que os índices de precisão teste-reteste do Teste de Goodenough aqui encontrados foram inferiores aqueles referidos pela própria Goodenough $(1926, r=0.937)$, porém próximos aos valores apresentados pela diversidade de índices comentados por Alves (1979, com índices de 0.65 a 0.957). Ou seja, mesmo utilizando um longo intervalo entre a primeira e a segunda testagem, as crianças apresentaram resultados finais similares, sinalizadores da estabilidade cognitiva conforme informações fornecidas pela maturidade conceitual expressa pelo DFH. Considerando, por sua vez, o índice de correlação obtido neste estudo entre teste-reteste com a EMMC $(r=0.586)$, o mesmo pareceu transcorrer. Comparando-o com os valores de correlação teste-reteste dos estudos originais americanos, entre 0.84 a 0.86 (Burgemeister, Blum \& Lorge, 1972) e aos índices obtidos no Brasil por Alves \& Duarte (1993, $r$ entre 0.73 e 0.90 ), notamos inferioridade nos valores encontrados no pre- sente estudo. Isto pode levantar a hipótese de que a maturidade conceitual avaliada por meio da EMMC poderia talvez ser mais sensível a fatores do desenvolvimento decorrentes dos seis meses de vida entre as avaliações realizadas no presente trabalho. Ou ainda que, o fato das crianças apresentarem dificuldades de aprendizagem, pode estar, de alguma forma, relacionado a menor estabilidade no desempenho cognitivo em atividades conceituais como as oferecidas pela EMMC. Certamente estas hipóteses estão sendo apresentadas neste momento como rascunhos iniciais de novas investigações, considerando-se 0 reduzido número de pesquisas sistematizadas sobre esta temática e com estes instrumentos no Brasil, embora freqüentemente utilizados na prática profissional.

Dentro das possibilidades informativas das atuais evidências empíricas obtidas com estes 41 escolares do ensino fundamental, no entanto, foi possível verificar a utilidade e atribuir razoável confiabilidade às avaliações psicológicas infantis por meio do Teste de Goodenough (1926/1964) e da EMMC (1972/1993) para a atual realidade sóciocultural brasileira, mesmo para crianças com dificuldades de rendimento escolar, foco de muitos dilemas na prática profissional cotidiana. Estes resultados procuraram constituir-se em estímulos atualizados para a reflexão e o aprimoramento técnico dos utilizadores destes testes psicológicos em nosso país, buscando contribuir para a melhoria da qualidade e das análises interpretativas das avaliações psicológicas realizadas na área do desempenho intelectual infantil.

\section{Referências Bibliográficas}

Abell, S.C., Horkheimer, R. \& Nguyen, S.E. (1998). Intellectual evaluations of adolescents via Human Figure Drawings: An empirical comparison of two methods. Journal of Clinical. Psychology, 54 (6), p. 811-815.

Aikman, K.G., Belter, R.W. \& Finch, A.J.Jr. (1992). Human Figure Drawings: Validity in assessing intellectual level and academic achievement. Journal of Clinical Psychology, 48 (1), p. 114120.

Alves, I.C.B. \& Duarte, J.L. (1993). M. Escala de 


\section{Susi Lippi Marques}

maturidade mental colúmbia: Manual para avaliação e interpretação. $3^{\mathrm{a}}$ ed., São Paulo: Casa do Psicólogo.

Alves, I.C.B. (1979). O Teste de Goodenough-Harris em uma população pré-escolar paulistana. Dissertação de Mestrado - Instituto de Psicologia de São Paulo - USP. São Paulo, 180 p.

Anastasi, A. (1976). Testes psicológicos. São Paulo: EPU.

Anastasi, A. \& Urbina, S. (2000). Testagem psicológica. Porto Alegre: Artes Médicas Sul.

Burgemeister, B.B., Blum, L.H. \& Lorge, I. (1993). Escala de Maturidade Mental Colúmbia: manual para aplicaçāo e interpretação. São Paulo: Casa do Psicólogo. Edição original: 1972.

Cunha, J.A. \& cols. (2000). Psicodiagnóstico-V. Porto Alegre: Artes Médicas Sul.

Goodenough, F. (1964). Test de inteligencia infantil por medio del dibujo de la figura humana. Buenos Aires: Paidos,. Edição original: 1926.

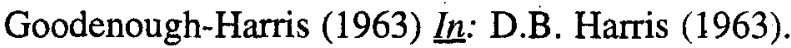
El test de Goodenough: Revisión, ampliación y actualización. Buenos Aires: Paidós.

Harris, D.B. (1963). El test de Goodenough: Revisión, ampliación y actualización. Buenos Aires: Paidós.

Hutz, C.S. \& Antoniazzi, S. (1995). O desenvolvimento do Desenho da Figura Humana em crianças de 5 a 15 anos de idade: Normas para avaliação. Psicologia: Reflexão e crítica, 8 (1), p. 318.

Santos, P.S., Colaço, N., Taborda, J. \& Alvarez, M. (1997). O Desenho da Figura Humana como técnica de avaliação psicológica: que qualidade, que limitações? In: M Gonçalves; I. Ribeiro; S Araújo; C. Machado; L. Almeida \& M. Simões (Org.). Avaliação Psicológica: Formas e contextos. Vol. V. Braga: APPORT.

Sarti, M.H.C. (1999). Estudo normativo dos itens evolutivos e indicadores emocionais de Koppitz $e$ índices de ansiedade de Handler em Desenhos de Figura Humana de escolares de Ribeirão Preto. Tese de Doutorado - Faculdade de Medicina de Ribeirão Preto - USP. Ribeirão Preto, 287 p.
Wechsler, S.M. (1996). O Desenho da Figura Humana: Avaliação do desenvolvimento cognitivo infantil. Campinas: Editorial Psy. 